\title{
Assessment of a Supervisory Fault-Hiding Scheme in a Classical Guidance, Navigation and Control Setup: the e.Deorbit mission
}

\author{
J. Cieslak ${ }^{1 \S}$, D. Henry ${ }^{1}$, P. Colmenarejo ${ }^{2}$, J. Branco ${ }^{3}$, N. Santis ${ }^{3}$, P. Serra ${ }^{3 £}$, J. Telaard ${ }^{4}$, H. Strauch ${ }^{4}$, \\ A.M. Giordanoi ${ }^{5}$, M. De Stefano ${ }^{5}$, C. Ott ${ }^{5}$, M. Reiner ${ }^{5}$, J. Jawarski ${ }^{6}$, E. Papadopoulos ${ }^{7}$, G. Visentin ${ }^{8}$, F. \\ Ankersen $^{8}$, J.G. Fernandez ${ }^{8}$
}

\begin{abstract}
The design of a model-based Fault Tolerant Control (FTC) strategy based on Virtual Actuators (VA) in a built-in Guidance, Navigation and Control (GNC) setup is addressed for the e.Deorbit space mission. This mission, initiated by the European Space Agency (ESA), aims at removing a large defunct satellite from Earth orbit: ENVISAT. The goal of this paper is to promote academic solutions to add fault tolerance capacities against thruster faults without any change or new tuning of the already in-place GNC solution. The validation of the proposed FTC solution is assessed by a simulation campaign based on a high-fidelity nonlinear industrial simulator.
\end{abstract}

\section{INTRODUCTION}

\section{A. Motivations}

According to [1], the number of orbital debris at the Low Earth Orbit (LEO) becomes critical. With the decreased costs to reach the space in the new programs, the debris population could inevitably grow and could lead to a rise of impractical orbital regions for space activities. In this context, the European Space Agency (ESA) has initiated a mission, entitled e.Deorbit, to remove a large (8.2tons, $26 \mathrm{~m} \times 10 \mathrm{~m} \times$ $5 \mathrm{~m})$ ESA-owned satellite from the LEO protected zone: ENVISAT. This motivated ESA to manage different debris removal solutions. One of them is based on a robotic arm, see Fig. 1. To this end, a joint academic and industrial research project named COMRADE (COntrol and Management of Robotics Active DEbris removal) has been initiated two years ago [2, 3]. The COMRADE study is limited to the development of solutions to the so-called Capture, Stabilization and Rigidisation phases of ENVISAT.

Since these phases are crucial ones for the success of the mission, some on-board abilities in terms of fault detection, isolation (FDI) and tolerant control (FTC) are required for the thruster-based chaser actuation system. This facet fitted with the fact that recent studies demonstrate that thruster faults account for approximatively one quarter of all Attitude and Orbit Control System (AOCS) failures [4], underlines a need of efficient FDI and FTC solutions to the mission fulfillment. The work addressed in this paper should be understood in this context.

${ }^{\S}$ Corresponding author, ${ }^{1}$ Authors are with the IMS-CNRS Lab. of Bordeaux University. E-mail: jerome.cieslak@ims-bordeaux.fr and david.henry@ims-bordeaux.fr; ${ }^{2}$ Author belongs to GMV Spain, pcolmena@gmv.com; ${ }^{3}$ Authors are with GMV Skysoft, Lisbon, Portugal (jbranco, nuno.santos,parroz@gmv.com); ${ }^{\mathfrak{E}}$ Author is involved in Lusofona University, Portugal; ${ }^{4}$ Authors are with Airbus Defence \& Space, Bremen, Germany (juegen.telaar,hans.strauch@airbus.com); ${ }^{5}$ Authors belongs to DLR e.V, Institute of Robotics and Mechnatronics, Wessling, Germany, (Alessandro.Giordano, Marco.DeStefano, Christien.Ott, Matthias.Reiner @ dlr.de); ${ }^{6}$ Author comes from to PIAP, Warsaw, Poland (jaroslaw.jaworski @pipa-space.com); ${ }^{7}$ Author is with NTUA Athens, Greece (egpapado@central.ntua.gr); ${ }^{8}$ Authors are with ESA/ESTEC, Noordwijk, Netherlands, (Gianfranco.Visentin, Finn.Ankersen, jesus.gil. fernandes@esa.int)

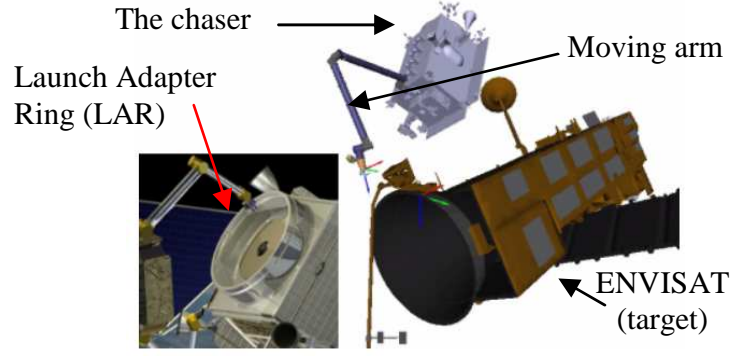

Figure 1. The ESA's e.Deorbit mission

\section{B. Antecedents and paper contribution}

In the literature, there exist a lot of academic papers dealing with the design of FTC solutions for spacecraft. Here, it is proposed to only focus on solutions used in real space missions. It appears that very few papers have been published to tackle the FTC problem dedicated to thruster faults. The main reason comes probably from to the historically use of hardware redundancy that leads to the development of reliable GNC setup with over-actuated feature [5, 6, 7].

Dealing with FTC solutions, Control Allocation (CA) solutions (see [8] for a survey) scheduled by a FDI unit, is the most retained. For instance, the work in [9] addresses the development of a CA-based FTC solution for flexible satellites. In $[10,11]$, the classical SIMPLEX-based CA algorithm is changed by the Nonlinear Inverse Pseudo Control (NIPC) solution to improve the FTC performances of the terminal rendezvous phase of the Mars Sample Return mission. An alternative solution can also be found in [12,13] by acting at the guidance level. The FTC strategy consists of the following principle: if the chaser is outside the so-called approach corridor or/and with an attitude outside of a predefined threshold, then a switch on a healthy redundant thruster set is made and a "retreat" maneuver is engaged. A new rendezvous trajectory is then planned by the guidance algorithm.

If it is possible to find some papers addressing the FTC problem for space debris removal missions $[14,15]$, it must outline that the only solution that has been assessed on an industrial high-fidelity benchmark considering the full problem (model of the avionics and actuators, propellant sloshing, non-spherical gravity acceleration, gravity gradient, solar radiation pressure, third-body perturbation (Moon and Sun), Earth magnetic disturbance and aerodynamic drag) is the one proposed in [16]. In this paper, a new class of nonlinear unknown input observer is proposed for the FDI solution. Fault tolerance is next achieved by means of the CA-based NIPC technique that is updated by FDI information.

This paper aims at proposing an alternative solution to the NIPC-based FTC technique. It is assumed that the FDI unit remains the same. More precisely, the proposed solution is based on the fault-hiding paradigm [17]. The motivation of 
using this technique as opposed to other existing ones, comes from the fact that the already in-place control loops (and especially their structure) that have been designed by the industrial partners of the project, are kept as they are, achieving de facto, the required robust performance when no fault occurs in the chaser's propulsion unit. The fault tolerance is obtained by inserting a reconfiguration block between the baseline control unit and the plant, see [18-21] to have some examples. The FTC principle consists of a reconfiguration unit - known under the name of Virtual Actuators (VA) - that hides the presence of a fault from the already in place controller. Of course, this reconfiguration unit is engaged if and only if a fault has been occurred and declared by the FDI unit.

The aim of this paper is thus to present the development and assessment of a VA-based FTC solution against thruster faults that may occur in the chaser spacecraft during rendezvous with ENVISAT. Note that the assessment is performed on the same high-fidelity nonlinear industrial benchmark used in [16] that is assumed to be fully representative of the mission. It is developed in Matlab/Simulink, within the library called SPACELAB that contains e.g. sensors, actuators, dynamic, kinematic, environment models and a universe library that provides the ephemerides of Earth, Moon, and Sun.

\section{PRoblem Statement}

In this study case, the considered reference scenario focus on the first part of the ENVISAT capture phase, i.e. the synchronization one. This phase starts with a transition from Parking Hold Point to Capture Point during which, the chaser synchronizes its motion with the target's movements until a position close enough so that the robotic arm can be deployed to capture ENVISAT at the grasping point located on the Launch Adapter Ring (LAR), see Fig. 1. As mentioned in the previous section, the proposed FTC solution consists in introducing VA units that will be in charge to hide the faults to the already in-place control law, see Fig. 2 for an illustration. Following the material background of the fault hiding paradigm [18-21], it is required a model of "what is seen from the VA unit" for its design. This is the purpose of the following sections ${ }^{1}$. Note that the derivation of this model is guided by the objective of designing the FTC scheme. In this sense, it is a simplified version of what has been implemented in the industrial benchmark.

\section{A. Modelling the chaser dynamics}

The chaser's model consists of its translational and rotational dynamics, considering all forces and torques acting on it. These dynamics are derived from the Cowell method (orbit dynamics and kinematic), the second Newton's law (rotational velocity) and the individual rotation matrices from Euler $(3,2,1)$ rotation (attitude). Forces and torques that are considered are those caused by propellant sloshing, the propulsion system (24 thrusters equip the chaser) and

\footnotetext{
1 Due to confidentiality reasons, we only discuss on the modeling principles, without any numerical values or deep details. The interested reader can however refer to [22] to more information.
}

disturbances. Propellant sloshing in tanks (two tanks equip the chaser) is modelled as a linear 3D spring-mass model that considers the Coriolis, the centrifugal, the Euler accelerations, the disturbances and of course, the forces and moments due to the actuation unit. The considered disturbances are central body acceleration, non-spherical gravity acceleration, gravity gradient, solar radiation pressure, third-body perturbation (Moon and Sun), the Earth magnetic field and the aerodynamic drag. Merging all these models into a unique state space, leads to a nonlinear state space representation that admits as inputs, forces $F_{s}(t)$ and torques $T_{s}(t)$ generated by the propulsion units and the above listed disturbances that we gather in a vector denoted $d(t)$. In terms of outputs, denoted $y(t)$, we consider the relative position and velocity, the chaser's angular rate and attitude. Then, considering that the relative velocity and the angular velocity are relatively small (which is the case for short range rendezvous mission), the nonlinear model can be approximated using a first order Taylor approximation. This leads to a linear state space representation of order 48 , with inputs $u=\left[\begin{array}{ll}F_{s}^{T} & T_{s}^{T}\end{array}\right]^{T}$, the disturbances $d$ and outputs $y$.

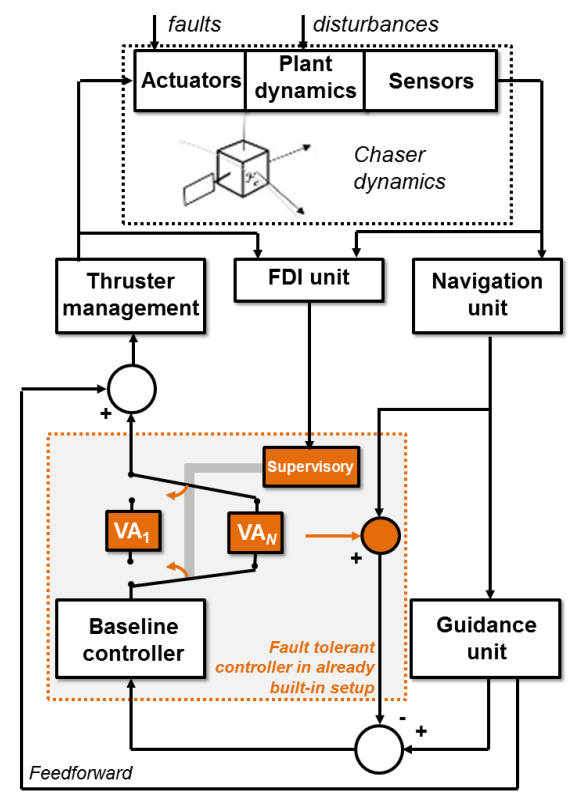

Figure 2. The proposed fault tolerant GNC setup

The so-derived model obviously depends on some uncertainties that mainly consist of the mass of the chaser, the propellant masses, and the modes of propellant sloshing (both in terms of frequencies and damping factors). To carry out these uncertainties, the LFT formalism commonly used in the $H_{\infty} / \mu$ control community, is used, see for instance [23]. This boils down to the following model of the chaser dynamics

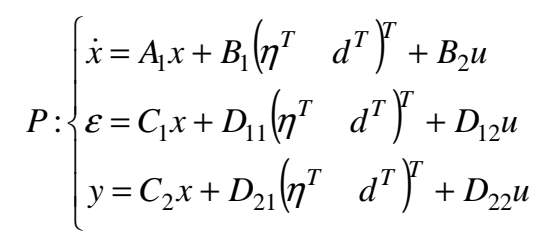

where $x \in \mathfrak{R}^{48 \times 1}, y \in \mathfrak{R}^{12 \times 1}, u \in \mathfrak{R}^{6 \times 1}$ and $d \in \mathfrak{R}^{6 \times 1} . \eta$ and $\varepsilon$ are internal signals and $\Delta$ is defined according to 


$$
\begin{aligned}
\Delta= & \operatorname{diag}\left(\delta_{m} I_{3}, \delta_{s 1 d} I_{9}, \delta_{s 1 f 1} I_{9}, \delta_{s 1 f 2} I_{9}, \delta_{s 1 f 3} I_{9}, \delta_{m 1} I_{3},\right. \\
& \left.\delta_{s 2 d} I_{9}, \delta_{s 2 f 1} I_{9}, \delta_{s 2 f 2} I_{9}, \delta_{s 2 f 3} I_{9}, \delta_{m 2} I_{3}\right): \delta_{k} \in \Re,\|\Delta\|_{\infty} \leq 1
\end{aligned}
$$

where $\delta_{m}, \delta_{m i}, \delta_{\text {sid }}, \quad \delta_{s i j}, i=\overline{1,2} j=\overline{1,3}$ refer to the mass uncertainty, the propellant masses, the damping factors and the frequencies of the sloshing modes, respectively.

\section{B. The baseline controller}

The chaser controller obeys to a feed-forward/feedback structure that issues force and torque commands to track the reference trajectories delivered by the guidance unit. The feed-forward is managed at the guidance level that is described later, in section II.F. The feedback controller has been designed using the $H_{\infty}$ mixed sensitivity approach [24]. This boils down to a linear controller robust against the uncertainties $\Delta$. Its state-space representation is given by

$$
\left\{\begin{array}{l}
\dot{x}_{c}=A_{c} x_{c}+B_{c}\left(y_{\text {ref }}-y\right) \\
u_{c}=C_{c} x_{c}+D_{c}\left(y_{\text {ref }}-y\right)
\end{array}\right.
$$

where $y_{\text {ref }} \in \mathfrak{R}^{12 \times 1}$ and $x_{c} \in \mathfrak{R}^{102 \times 1}$ are the reference signal to track and the controller state vector, respectively. $A_{c}, B_{c}, C_{c}$ and $D_{c}$ are real matrices of appropriate dimensions.

\section{The thruster management unit}

The thruster management unit is nothing else than a control allocation algorithm used to convert the force and torque commands $u_{c}(t)$ (see Eq. (2)), into thruster commands $u_{T H R}(t)$. In this study case, the engineers proposed to retain the SIMPLEX-based algorithm [8]. Note that the spacecraft possesses 24 thrusters of $22 \mathrm{~N}$ to control both attitude and position motions. Let us introduce the thruster configuration matrix $\mathrm{M}_{\mathrm{THR}} \in \mathfrak{R}^{6 \times 24}$. The elements of $\mathrm{M}_{\mathrm{THR}}$ are the influence coefficients defining how each thruster affect each component of $u$ in (1), i.e.

$$
\mathrm{M}_{\mathrm{THR}} u_{T H R}=u_{c}
$$

Then following $[12,16]$, a useful model of the thruster management unit consists of the left pseudo-inverse of $\mathrm{M}_{\mathrm{THR}}$ so that $\mathrm{M}_{\mathrm{THR}}{ }^{+} u_{c}=u_{T H R}$, where $(\bullet)^{+}$denotes the left pseudo-inverse of matrices.

\section{The FDI unit}

The FDI unit is based on the technique described in [16] that consists of a new class of NonLinear Unknown Input Observers (NL-UIO) that is optimal in the $L_{2}$-gain sense. The interested reader can refer to [16] to have a clear overview of this solution, from the fault isolability discussion to the NLUIO design.

\section{E. The navigation unit}

It is important to recall that for this space mission, ENVISAT (the target) will be considered like a passive target, i.e. the ENVISAT's actuators, sensors and its telemetry system cannot be used. Hence, the retained avionic architecture for the chaser is composed of a Light Detection and Ranging (LIDAR) unit which allows having the full relative pose estimation, an Inertial Measurement Unit
(IMU), 3 Star Tracker heads, a Sun Sensor, a Global Positioning System (GPS) receiver and a GPS constellation propagation. Hence, the navigation units provides the current situation of the chaser both relative to the target, (relative position and velocity) and its attitude and angular velocity. We recall that these last measures are those associated with the model (1).

\section{F. The guidance unit}

The feed-forward actions and trajectory reference profile mainly consist of a classic rendezvous trajectory (forced translation while keeping a constant attitude) and a spin synchronization trajectory, see Fig. 3 for an illustration.

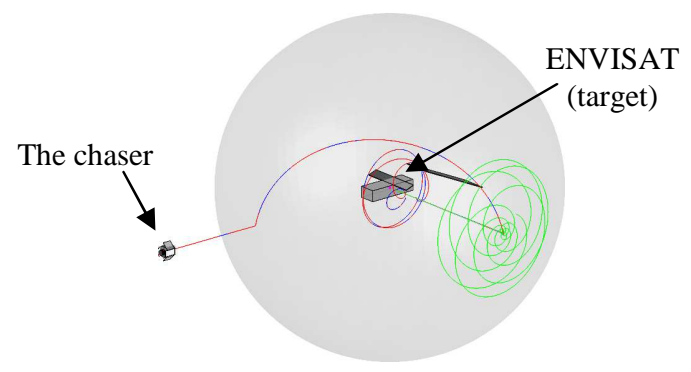

Figure 3. The synchronisation trajectory

\section{G. Problem formulation}

We are now ready to formulate the FTC design problem. Fig. 4 shows in details how the proposed FTC solution operates at the GNC level. The goal we pursue is to design the virtual actuator units, to cover any type of fault occurring in the thrusters. Following the method proposed in [18-21], we need to design a total of 25 VA units, one (the first, $i=1$ ) is devoted to the fault-free case and the others $(i=\overline{2,25})$ are dedicated to faults that may occur in the 24 thrusters. The control signal applied to the plant will be now $u_{f_{i}}, i=\overline{1,25}$ (defined later) instead of $u_{c}$. According to [18-21], all VA units can be designed under the assumption of no uncertainty. Indeed, the baseline controller will be able to manage correctly the uncertainties when the fault hiding paradigm succeeds. Finally, and as it is extensively discussed in [1821], the fundamental problem of the control signal saturation that may occur due to the presence of a fault, can be managed in this setup belonging to the fault-hiding paradigm. To taking into account all the previous aspects, the model (1) is reformulated for the $i^{\text {th }}$ operating mode according to:

$$
\left\{\begin{array}{c}
\dot{x}=A x+B_{i} \operatorname{sat}\left(u_{f_{i}}\right) \\
y=C x
\end{array}, B_{i}=B W_{i} \mathrm{M}_{\mathrm{THR}}\right.
$$

where $W_{i}=\operatorname{diag}\left(w_{k i}\right), k=\overline{1,24}$ is a diagonal matrix so that the fault-free situation corresponds to $W_{i}=I_{24 \times 24}$ If the $k^{\text {th }}$ element $w_{k i}$ is equal to 0 , this means that the $k^{\text {th }}$ actuator is out of order. The function $\operatorname{sat}\left(u_{f_{i}}\right)$ is defined according to:

$$
\operatorname{sat}\left(u_{f_{i}}\right)=\left(\begin{array}{c}
\operatorname{sat}\left(u_{f_{i 1}}\right) \\
\vdots \\
\operatorname{sat}\left(u_{f_{i 6}}\right)
\end{array}\right), \operatorname{sat}\left(u_{f_{i 1}}\right)=\left\{\begin{array}{cl}
\alpha_{i 1} & \left(u_{f_{i 1}}>\alpha_{i 1}\right) \\
u_{f_{i 1}} & \left(\left|u_{f_{i 1}}\right| \leq \alpha_{i 1}\right) \\
-\alpha_{i 1} & \left(u_{f_{i 1}}<-\alpha_{i 1}\right)
\end{array}\right.
$$




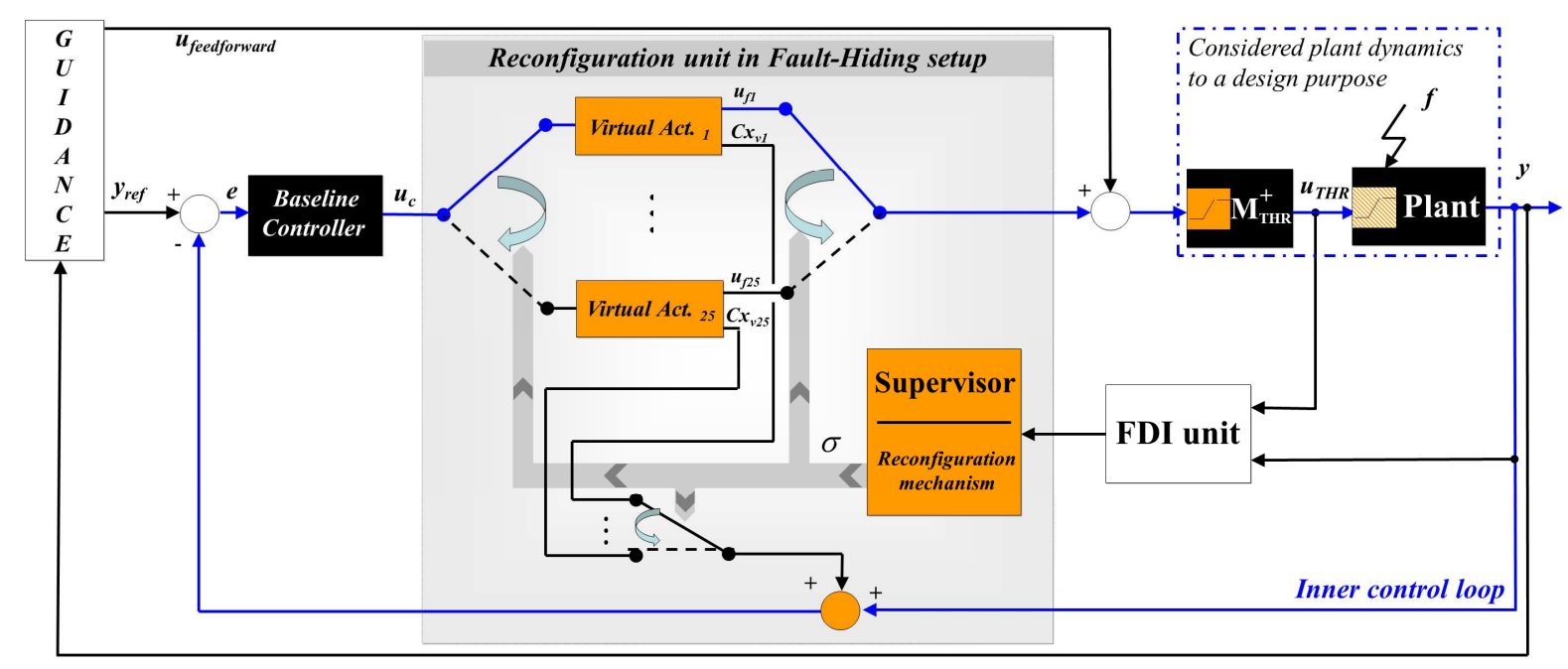

Figure 4. Fonctional diagram to formulate the FTC design problem

From (4) and Fig. 4, it can be seen that it is proposed to relocate the physical input saturations in input constraints on the desired forces and torques. This is why the saturation function of Fig. 4 is now put on the input signal of the thruster management unit. With this setup, the signal $u_{c}$ of (3) is now replaced by the fault tolerant control signal $u_{f_{i}} \in \mathfrak{R}^{6 \times 1}, i=\overline{1,25}$. This signal is provided by the $i^{\text {th }} \mathrm{VA}$ unit (see Fig. 4) that has been designed to the $i^{\text {th }}$ mode, from a bank of pre-computed VAs defined according to

$$
\left\{\begin{array}{l}
\dot{x}_{v_{i}}=\left(A+B_{i}^{*} M_{i}\right) x_{v_{i}}+\left(B_{1}-B_{i}^{*}\right) u_{c} \\
u_{f_{i}}=N_{i} u_{c}-N_{i} M_{i} x_{v_{i}}
\end{array}, i=\overline{1,25}\right.
$$

where $M_{i} \in \mathfrak{R}^{6 \times 12}$ are the virtual actuator gains to be designed such that $\left(A+B_{i}^{*} M_{i}\right)$ is Hurwitz. The matrices $N_{i}$ and $B_{i}^{*}$ are obtained by:

$$
\begin{gathered}
N_{i}=\left(B_{i}^{*}\right)^{+} B_{1} \\
B_{i}^{*}=B_{i} N_{i}=B_{i}\left(B_{i}^{*}\right)^{+} B_{1}
\end{gathered}
$$

Following the notations introduced previously for the model (4), $B_{1}$ is the fault-free matrix of (4), i.e. $B_{1}=B \mathrm{M}_{\mathrm{THR}}$. From Fig. 4 , it can be noticed that the output of fault tolerance unit is given by $y+C x_{v_{k}}$ where $x_{v_{k}}$ corresponds to the state of the selected virtual actuator.

Remark 1: In fault-free situation, the first VA is inserting in the closed-loop. This situation doesn't degrade the control performances achieved by the baseline controller since $\left(B-B_{1}^{*}\right)$ is equal to zero. By choosing $x_{v_{1}}(0)=0$ and since $\left(A+B_{1}^{*} M_{1}\right)$ is Hurwitz, we directly applied the control signal $u_{c}$ to the plant. This setup has been proposed to have a setup able to deal with the case of intermittent fault.

Once a fault has been diagnosed by the FDI algorithm [16], a switching mechanism is engaged to select the adequate VA unit. Here, the switching algorithm that is proposed is based on the supervisor concept [21], [26], [27]. In this approach, the switching logic is a decision map that generates a piecewise constant switching signal $\sigma(t)$. The problem can thus be formulated according to:
Problem 1: Let the baseline controller (2), the model (4) that covers the 25 operating modes with the associated VA unit given by (6)-(8) be considered. Let the following control law be introduced

$u=\left\{\begin{array}{ll}u_{f_{1}} & t<t_{s} \\ u_{f_{i}} & t \geq t_{s}\end{array}, i=\overline{1,25}\right.$

where $t_{s}$ is the fault occurrence time declared by the FDI module described in section II.D. The goal we pursue is to design $u_{f_{i}} i=\overline{1,25}$ subjected to input constraints (5) by means of the $i^{\text {th }}$ virtual actuator (6) and the model (4) such that $\left(A+B_{i}^{*} M_{i}\right)$ is Hurwitz.

\section{SOLUTION TO THE PROBLEM}

Before starting the FTC design, it is natural to ask about the ability of the spacecraft to fulfill its mission in spite of a thruster fault. To answer this question, a preliminary study based on [28] can be performed. The following can be considered if and only if the faulty situation is recoverable. A solution to Problem 1 is given by the following theorem.

Theorem 1: For each operating mode $i=\overline{1, Q}$ with $Q=25$, let the following design problem be considered $Q$ times to obtain $Q$ virtual actuator gains. Let $X_{v a_{i}}^{-1}, P_{i}$ and $\Gamma_{i}$ be defined such that

$$
\begin{aligned}
& H e\left\{\left(\begin{array}{cc}
v_{f_{i}} A_{c l} P_{i}^{-1} & 0 \\
v_{f_{i}} A_{i}^{*} P_{i}^{-1} & A X_{v a_{i}}{ }^{-1}+B_{i}^{*} \Gamma_{i}
\end{array}\right)\right\} \prec 0 \\
& \left(\begin{array}{cc}
X_{v a_{i}}^{-1} & \left(\Gamma_{i}^{(j)}\right)^{T} \\
\Gamma_{i}^{(j)} & \frac{\left(\frac{\alpha_{i k}}{\left\|N_{i}^{(k)}\right\|}-\mu_{f_{i}}\right)^{2}}{n_{\tilde{u}_{i}}}
\end{array}\right) \succ 0 \quad k=\overline{1,6} \quad\left\|N_{i}^{(k)}\right\| \neq 0
\end{aligned}
$$

hold where:

$$
\begin{aligned}
& A_{c l}=\left[\begin{array}{cc}
A-B_{1} D_{c} C & B_{1} C_{c} \\
-B_{c} C & A_{c}
\end{array}\right] \\
& A_{i}^{*}=\left(\begin{array}{ll}
-\left(B_{1}-B_{i}^{*}\right) D_{c} C & \left(B_{1}-B_{i}^{*}\right) C_{c}
\end{array}\right)
\end{aligned}
$$




$$
\mu_{f_{i}}=\max _{e\left(P_{i}, v_{f_{i}}\right)}\left\|u_{c}\right\|
$$

$N_{i}{ }^{(k)}$ is the $k^{\text {th }}$ row of $N_{i}$. In the same spirit, $\Gamma_{i}^{(j)}$ denotes the $j^{\text {th }}$ row of $\left.\left.\Gamma_{\dot{d}}, v_{f_{i}} \in\right] 0,1\right] . n_{\widetilde{u}_{i}}$ is the number of non-zero elements in $N_{i}^{(k)}$ and $j$ in (10) takes values corresponding to the indices of the non-zero elements in $N_{i}{ }^{(k)} . H e\{M\}$ is equal to $M+M^{T}$. Then, the virtual actuator gain $M_{i}$ obtained by $M_{i}=\Gamma_{i} X_{v a_{i}}$ will guarantee that the plant (4) controlled by (2) and (6) is contractively invariant and respect the input constraints given in (5).

Proof: The proof of Theorem 1 is mainly based on the one developed in [19]. Here, it is just shown that the solution is still valid even if the baseline controller contains integrators (which is the case for (2)) and when the state vector dimension of the controller (2) and the plant (4) differ (which is often the case when a controller is designed using a $H_{\infty} / \mu$ synthesis technique). To proceed, let the control loop composed by (2), (3), (4) and (6) be considered without including (5). When the operating mode is well identified, the closed-loop can be put in a block-triangular form by introducing the variable $x_{w_{i}}=x_{r}+x_{v_{i}}$. It follows that the state-space representation can be written according to

$$
\left(\begin{array}{c}
\dot{x}_{c l_{i}} \\
\dot{x}_{v_{i}}
\end{array}\right)=\left(\begin{array}{cc}
A_{c l} & 0 \\
A_{i}^{*} & A+B_{i}^{*} M_{i}
\end{array}\right)\left(\begin{array}{c}
x_{c l_{i}} \\
x_{v_{i}}
\end{array}\right)+\left(\begin{array}{c}
B^{y} \\
\left(B_{1}-B_{i}^{*}\right) D_{c}
\end{array}\right) y_{r e f}
$$

where:

$$
x_{c l_{i}}=\left(\begin{array}{c}
x_{w_{i}} \\
x_{c}
\end{array}\right), B^{y}=\left(\begin{array}{c}
B_{1} D_{c} \\
B_{c}
\end{array}\right)
$$

Assuming that $y_{\text {ref }} \in L_{\infty}^{y_{\text {ref }}}$ is exogenous and bounded, the stability of the closed-loop involves the stability of:

$$
\left(\begin{array}{c}
\dot{x}_{c l_{i}} \\
\dot{x}_{v_{i}}
\end{array}\right)=\left(\begin{array}{cc}
A_{c l} & 0 \\
A_{i}^{*} & A+B_{i}^{*} M_{i}
\end{array}\right)\left(\begin{array}{l}
x_{c l_{i}} \\
x_{v_{i}}
\end{array}\right)
$$

Let the following Lyapunov function be considered

$$
V_{2_{i}}(t)=\left(\begin{array}{c}
x_{c l_{i}}(t) \\
x_{v_{i}}(t)
\end{array}\right)^{T}\left(\begin{array}{cc}
\frac{P_{i}}{v_{f_{i}}} & 0 \\
0 & X_{v a_{i}}
\end{array}\right)\left(\begin{array}{c}
x_{c l_{i}}(t) \\
x_{v_{i}}(t)
\end{array}\right)
$$

where $X_{v a_{i}}$ is a symmetric positive definite matrix. To guarantee $V_{2_{i}}<0$, it is necessary to verify

$$
H e\left\{\left(\begin{array}{cc}
\frac{P_{i}}{v_{f_{i}}} & 0 \\
0 & X_{v a_{i}}
\end{array}\right)\left(\begin{array}{cc}
A_{c l} & 0 \\
A_{i}^{*} & A+B_{i}^{*} M_{i}
\end{array}\right)\right\}<0
$$

that is a Linear Matrix Inequality (LMI) equivalent to (9) by introducing $\Gamma_{i}=M_{i} X_{v a_{i}}^{-1}$. Hence, the closed-loop is asymptotically stable by taking into account (9) in a LMI problem. Now, let the discharge on input constraints be now removed to complete the proof. Following the procedure introduced in [19], it is possible to show the validity of theorem 1. Since the procedure is similar to [19], the remaining of the proof is not explicitly given here. The interested reader can refer to [19] for more details.
Theorem 1 provides the conditions to design the gain of virtual actuators in order to maintain the closed-loop system state inside a stable region as long as this state belongs to this region at the fault isolation time $t_{s}$. Hence, the state trajectory will converge, despite the change of the matrix $B$ due to the fault occurrence and input constraints (5).

\section{NONLINEAR SIMULATION CAMPAIGN}

Independently of the FTC solution, it is fundamental to guarantee that the available actuator resources are sufficient to maintain the control objectives despite the presence of a thruster fault. This is refers to the fault compensability property. This problem is formulated in [28] using the socalled attainable force/torque domains. Applying this technique to our problem, the result revealed that it is possible to fulfill the e.Deorbit capture mission in the case where one thruster is out of order. Two other key features in the theory presented in section III can be extracted:

- the determination of the parameters $\alpha_{i k}, k=\overline{1,6}$ for all $i=1,25$ involved in (5) that fundamentally represent the maximum attainable force/moment in the $x, y, z$ directions, under fault-free and faulty situations. This problem has been solved using the attainable force/torque domains analysis technique;

- the determination of the parameters $\mu_{f_{i}} \quad i=\overline{1,25}$ that enter in (10) and represent the maximum magnitude of $u_{c}$ according to (13). These parameters have been determined through several simulations in healthy situations.

The VA units are implemented within the industrial highfidelity simulator of the e.Deorbit. Figures 5 and 6 show the obtained results when the thruster 1 is stuck in open position. The same simulation has been performed when the already in-place GNC setup works in healthy (green line) and faulty (dotted red line) situations, and when the proposed solution has been integrated (blue line). If the improvement for the relative position error is mainly related to the time necessary to recover the nominal (no fault) situation (see Fig. 6), one can noticed an improvement of $45 \%$ on the transient attitude errors after the fault occurrence, see Fig. 5.

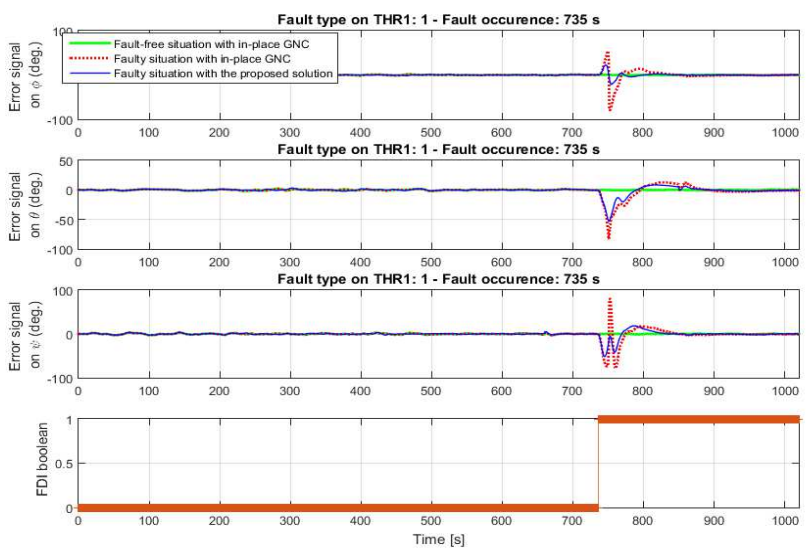

Figure 5. Attitude errors for a fault in the thruster 1 

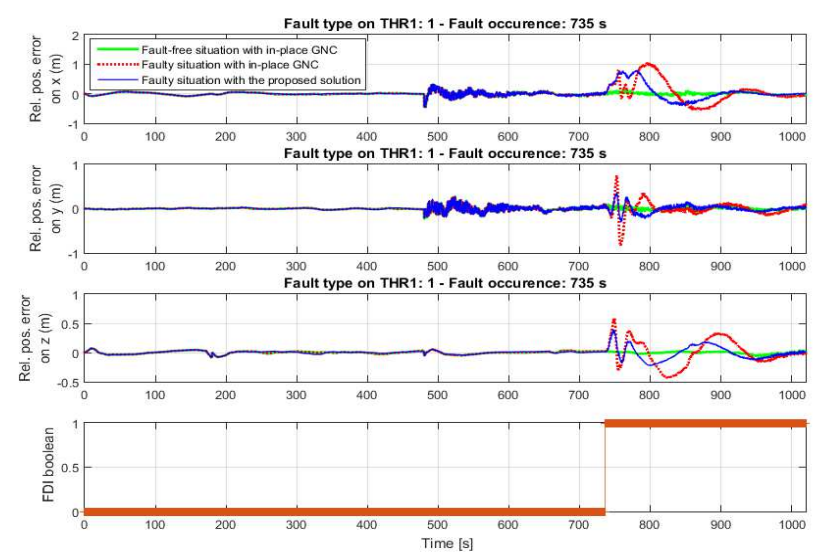

Figure 6. Relative position errors for a fault in the thruster 1

A simulation campaign is next conducted for each each thruster fault type (stuck opened and stuck closed) and in fault free situations. Due to place limitation, only the cases of faults occurring in thrusters $1,5,13$ and 17 are presented. A total of 60 runs have been performed, 10 in healthy situations and 50 in faulty ones. During this campaign, the fault time occurrence is randomly chosen. Figures 7 and 8 illustrate the behavior of the attitude (in degree) and relative position (in meter) errors for the case of stuck-opened and stuck-closed faults. The fault-free runs have been superposed (red plots) for a better illustration of the performance of the proposed FTC solution. It can be seen that we obtain transient deviations similar to the ones show in Fig. 5 and 6 , which demonstrates the potential of the approach. Finally, we argue that since the attitude and relative position during the last $100 s$ of the rendezvous with ENVISAT are within their required values, the mission is not in danger despite the presence of the fault. Thus, the robotic arm can be deployed and the capture of ENVISAT can be performed. This last point definitively promotes the relevance of the proposed FTC solution.
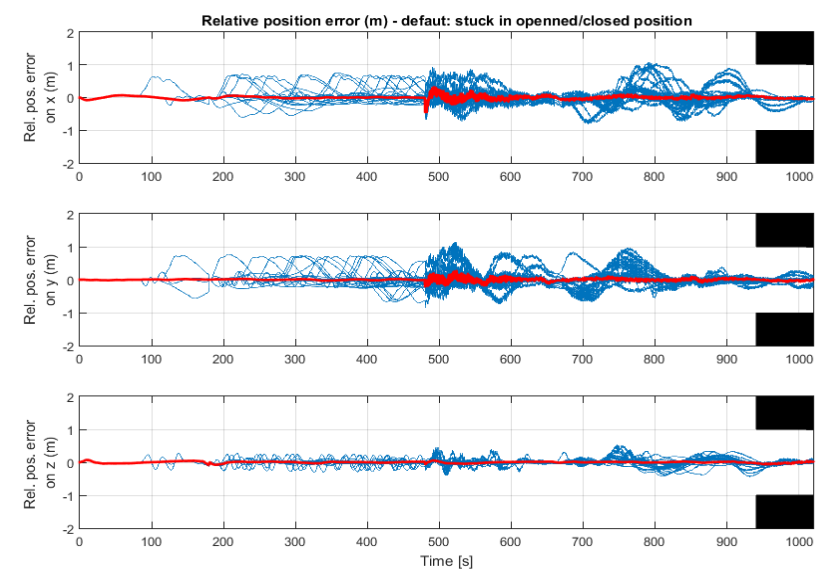

Figure 7. Relative position errors in a Monte Carlo campaign

\section{CONCLUSION}

In this paper, a model-based FTC strategy against thruster faults has been presented for the ESA's e.Deorbit mission whose aim it to remove the satellite ENVISAT from the Earth orbit. It is shown that the proposed FTC scheme can be integrated in an already built-in GNC setup and tuned to achieve specific objectives in nominal (no fault) situations. The proposed solution is based on the fault-hiding paradigm and the design of virtual actuator gains has been performed to respect input constraints. A simulation campaign based on a high-fidelity industrial benchmark highlights the efficiency of the proposed solution. Future works will be required to assess this technique for a less actuated system to better appreciate its benefit.
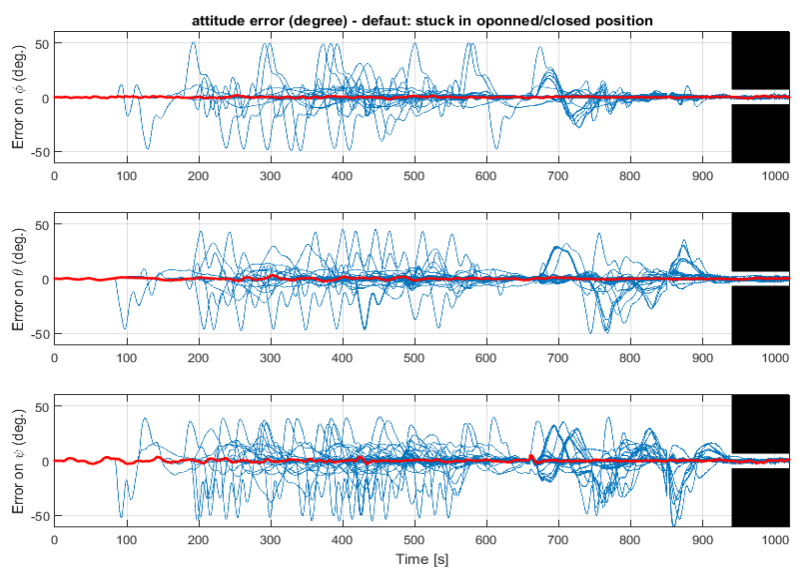

Figure 8. Attitude errors in a Monte Carlo campaign

\section{REFERENCES}

[1] J.-C. Liou \& N. L. Johnson, "Instability of the present LEO satellite populations", Advances in Space Research, vol. 41(7), pp. 10461053, 2008

[2] P. Colmenarejo, J. Telaar, C. Ott, J. Branco, J. Jarowski, E. Papadopoulos, D. Henry, G. Visentin. "COMRADE: Control and Management of Robotics Active Debris Removal." Clean Space Industrial days, 24-27 October 2017. ESTEC. Netherlands.

[3] P. Colmenarejo, J. Branco, N. Santos, P. Arroz, J. Telaar, H. Strauch, C. Ott, M. Reiner, D. Henry, J. Jaworski, E. Papadopoulos, G. Visentin, F. Ankersen, J. Gil-Fernandez. "Methods and outcomes of the COMRADE project - Design of robust coupled control for robotic spacecraft in servicing missions". Int. Astronautical Congress, Oct 2018, Bremen, Germany.

[4] M. Tafazoli, "A study of on-orbit spacecraft failures". Acta Astronautica, 64, 195-205, 2009.

[5] Passot, X., Cussac, T., Mouret, J.-M., Carayon, J.-L., Laffaye, C., Cier, P.-A, "The Myriade Micro-Satellite Computer and Software: A Reliable System Based on a Low Cost Computer", Data Systems in Aerospace, 2 June, 2005 in Edinburgh, Scotland

[6] Butler R.W., "A Primer on Architectural Level Fault Tolerance", NASA/TM-2008-215108, 2008

[7] Wander A. \& Förstner R., "Innovative fault detection, isolation and recovery strategies on-board spacecraft: state of the art and research challenges", Deutscher Luft- und Raumfahrtkongress, 2012

[8] Johansen, T.A. and Fossen, T.I., "Control allocation: a survey". Automatica, 49, 1087, 2013

[9] Fu, Y.P., Cheng, Y.H., Jiang, B., Yang, M.K., "Fault tolerant control with on-line control allocation for flexible satellite attitude control system”, IEEE Int. C. on Intelligent Contr. and Inf. Processing, 2011.

[10] R. Fonod, D. Henry, C. Charbonnel, E. Bornschlegl, D. Losa, et al., "Robust FDI for fault-tolerant thrust allocation with application to spacecraft rendezvous", Contr Engineering Practice, 2015, 42, 12-27.

[11] R Fonod, D Henry, C Charbonnel, E Bornschlegl , "A Class of Nonlinear Unknown Input Observer for Fault Diagnosis: Application 
to Fault Tolerant Control of an Autonomous Spacecraft", 10th UKACC International Conference on Control, 19-24, 2014

[12] D Henry, C Le Peuvédic, L Strippoli, F Ankersen, "Model-based FDIR and fault accommodation for a rendezvous mission around the Mars planet: the Mars sample return case", IFAC-PapersOnLine 49 (5), 266-271, 2015

[13] C Le Peuvédic, C Charbonnel, D Henry, L Strippoli, F Ankersen, "Fault tolerant control design for terminal rendezvous around mars", 9th Int. ESA Conference on GNC Systems, 2014

[14] Cordeiro D., "Fault-Tolerant Control for Terminal Rendezvous inActive Removal of Space Debris", Master of Science Degree, Universidade de Lisboa, 2017

[15] Biondi G., Mauro S., Pastorelli S., Sorli M., "Fault-tolerant featurebased estimation of space debris rotational motion during active removal missions", Acta Astronautica, 2018

[16] Henry D., Cieslak J., Zenteno Torres J., Colmenarejo P., Branco J., et al, "Model-based fault diagnosis and tolerant control: the ESA's e.Deorbit mission", IEEE European Control Conference, Italy, 2019

[17] Lunze J. \& Steffen T., 'Control reconfiguration after actuator failures using disturbance decoupling methods,' IEEE Trans. Autom. Control, 51 (10), 2006, pp. 1590-1601.

[18] Seron M.M., De Donà J.A., Richter J.H., 'Integrated sensor and actuator fault-tolerant control', Int. J. of Control, 86(4), 2013.

[19] Rotondo D., Ponsart J.C., Theilliol D., Nejjari F., Puig V., 'A virtual actuator approach for the fault tolerant control of unstable linear systems subject to actuator saturation and fault isolation delay', Annual reviews in Control, 39, 68-80, 2015.

[20] Rotondo D., Puig V., Nejjari F., Romera J., 'A fault-hiding approach for the switching quasi-LPV Fault-tolerant control of a four-wheeled omnidirectional mobile robot', IEEE trans. Indus. Electronics, 62 (6), 3932-3944, 2015.

[21] Cieslak J. \& Henry D., "A Switching Fault-Hiding Mechanism based on Virtual Actuators and Dwell-time Conditions", IFAC Safeprocess'18, Warsaw, Poland, 2018.

[22] D. Henry, F. Ankersen, L. Strippoli, "A class of Unknwon Input Observers under $H_{\infty}$ performance for Fault Diagnosis: Application to the Mars Sample Return mission", Chapter in New Trends on Observer based Controller Design and their Applications, Eds Elsevier, 2019.

[23] Cockburn, J., Morton, B., 1997. Linear fractional representations of uncertain systems. Automatica 33 (7), 1263-1271.

[24] Doyle, J.C., Glover, K., Khargonekar, P., and Francis, B., 'State-space solutions to standard $\mathrm{H}_{2}$ and $\mathrm{H}_{\infty}$ control problems', IEEE Trans. Aut. Control, 1989, vol 34, ${ }^{\circ} 8$, pp. 831-847

[25] Preda V., Cieslak J., Henry D., Bennani S., Falcoz A., "Robust microvibration mitigation and pointing performance analysis for high stability spacecraft", Int. Journal of Robust and Nonlinear Control, 2018, 28 (18), pp.5688-5716

[26] Hespanha J.P., Liberzon D., Morse A.S., "Hysteresis-Based Supervisory Control of Uncertain Linear Systems", Automatica, 2003

[27] Efimov D., Cieslak J., Henry D. 'Supervisory fault-tolerant control with mutual performance optimization', Int J. of Adapt. Contr .and Signal Processing, 27, pp.251-279. 2013

[28] Zenteno Torres J., Cieslak J., Henry D., Davila J., "Fault Compensability Criteria with Application to a Rendezvous Mission Around Mars", 14th International Workshop on Advanced Control and Diagnosis, 2017 